\title{
Triangle and concave pentagon electrodes for an improved broadband frequency response of stripline beam position monitors
}

\author{
Yoshihiro Shobuda \\ JAEA, Japan Atomic Energy Agency, 2-4 Shirakata, Tokaimura, Nakagun, Ibaraki 319-1195, Japan \\ Yong Ho Chin, Koji Takata, and Takeshi Toyama \\ KEK, High Energy Accelerator Research Organization, 1-1 Oho, Tsukuba, Ibaraki 305-0801, Japan \\ Keigo Nakamura \\ Kyoto University, Kitashirakawa, Oiwake-cho, Sakyo-ku, Kyoto 606-8502, Japan
}

(Received 19 September 2015; published 29 February 2016)

\begin{abstract}
The frequency domain performance of a stripline beam position monitor depends largely on the longitudinal shape of its electrode. Some shapes other than a conventional rectangle have been proposed and tested. To attain a good impedance matching along the electrode, they need to be precisely bent down toward their downstream in proportion to their width. This is a considerable task, and a failure to comply with it will result in a large distortion of the frequency-domain transfer function from the ideal one due to unwanted signal reflections. In this report, we first propose a triangle electrode for easy fabrication and setup: it only requires that a triangularly cut flat electrode will be placed in a chamber while being obliquely inclined toward the downstream port. Theoretical and simulation results show that the simple triangle electrode has a remarkably flatter frequency response than the rectangle one. The frequency response, in particular at high frequencies, can be further improved by attaching an "apron" plate, perpendicular to the upstream edge of the electrode. The overshooting of the frequency response at low frequency can be eliminated by replacing the straight sidelines of the triangle by three-point polylines (with a result that the triangle is transformed to a concave pentagon). The concave pentagon electrode needs to be bent only once at the middle point of the polylines for a good impedance matching and thus its fabrication and setup remain to be easy. Rf measurements for the various electrode shapes have been carried out. We found that the concave pentagon electrode achieves a wide and flat frequency response up to about $4 \mathrm{GHz}$ for the J-PARC Main Ring (MR).
\end{abstract}

DOI: 10.1103/PhysRevAccelBeams.19.021003

\section{INTRODUCTION}

In the Japan Proton Accelerator Research Complex (J-PARC) [1] Main Ring (MR), transverse intrabunch oscillations have been observed with a result of particle losses during the injection and at the onset of acceleration [2]. In order to suppress the beam instabilities, a wide-band (about $100 \mathrm{MHz}$ ) intrabunch feedback system has been installed [3]. Stripline beam position monitors (SBPM), operated at $108.8 \mathrm{MHz}$, are used to detect the transverse positions of the beam. There is a concern that electron cloud instabilities may appear and limit the beam current at future higher power operation [4]. To prepare for that case, we started to develop a wider-band (several $\mathrm{GHz}$ ) beam position monitor.

Published by the American Physical Society under the terms of the Creative Commons Attribution 3.0 License. Further distribution of this work must maintain attribution to the author(s) and the published article's title, journal citation, and DOI.
Stripline and slotted pickups $[5,6]$ are frequently used as both kickers and pickups for such feedback system. The latter tends to be used for stochastic cooling [7]. Cesaratto et al. [8] studied three types of transverse kickers (a stripline kicker, a cavity kicker, and a slotted kicker) for the Super Proton Synchrotron (SPS) at CERN. By optimizing the size parameters such as the slot spacing, the length of slotted section etc., the slotted-coaxial kicker is able to have an uniform frequency characteristic up to at least $1 \mathrm{GHz}$, while it has a broadband beam coupling impedance (for reference, the coupling impedance of a stripline electrode is shown in Appendix A.).

In J-PARC MR, due to the space limit, the use of the existing SBPM is desirable for detecting the electron cloud instabilities. But, the present SBPMs have rectangular electrodes. They have a comb-shape frequency response, like the red line in Fig. 1, far from a broad and flat response.

To replace the rectangular electrodes, we have proposed in a previous paper [9] an electrode with polynomial shape in the longitudinal direction, for a wide and flat frequency response. Theoretically, this shape seems to have the best 
frequency performance. However, actual measurements showed rather disappointing results. There are two conflicting requirements for a wide and flat frequency response. One is that the electrode shape needs to be smoothly tailored toward its tip, and thus it tends to become a quite complicated shape. Another one is that the electrode needs to be precisely bent down toward its tip, in proportion to their width, to attain a good impedance matching along the electrode. Failure to comply with the second requirement can easily make void of the merit of the first one.

Before our proposal of the polynomial electrode, Linnecar has proposed the exponential electrode for SPS at CERN [10]. For signals below $2 \mathrm{GHz}$, it has a frequency characteristic with about $35 \%$ peak to peak modulation of the magnitude response [10]. The same type of exponential electrodes were installed in the J-PARC MR. The J-PARC exponential electrodes have a frequency characteristic with about $30 \%$ peak to peak modulation of the magnitude response, below $1 \mathrm{GHz}$ [2,11] (See Fig. 24.). We have learned that the final frequency performance of electrode strongly depends on how precisely the electrode is fabricated and set up, which are quite difficult for electrodes with a complicated shape, such as exponential and polynomial.

From these experiences, we concluded that we should take a different approach, to start with a simple shape, easy to fabricate and set up, and gradually increase its complexity to improve the frequency performance. We anticipate that the frequency performance will be saturated quickly as the complexity of the shape is increased, since the potential merit by a sophisticated shape will be offset by the difficulty in compliance of the second requirement.

We first review the frequency performance of Linnecar's exponential electrode in Sec. II. We then show in Sec. III that a simple triangle electrode has the frequency performance as good as the exponential electrode. Simulations show that the attachment of an apron plate perpendicular to the upstream edge of the electrode can largely improve degradation of the frequency response at high frequency. The overshooting of the frequency response at low frequency can be eliminated by replacing the straight sidelines of the triangle by three-point polylines (i.e. the triangle is deformed to a concave pentagon.). In Sec. IV, we show measurements results that confirm the concave pentagon electrode with the apron has a good frequency response up to about $4 \mathrm{GHz}$ for the J-PARC MR. The paper is concluded in Sec. V.

\section{FREQUENCY PERFORMANCE OF THE EXPONENTIALLY TAPERED ELECTRODES}

Let us review Linnecar's exponential electrode first. For any shape of electrode, its characteristic impedance should be kept constant along the longitudinal direction. Both ports of the electrodes should be terminated by resistors with the same value as the characteristic impedance of the electrode.
Based on the two-dimensional theory, the beam inducedvoltage at the upstream port is proportional to the transfer function $F(\omega)$ [12] (See Appendix B.). The transfer function $F(\omega)$ for the electrode of length $l$ is analytically given by [12]

$F(\omega)=i \omega \int_{0}^{\frac{2 l}{c}} \frac{1}{2} k\left(\frac{c t}{2}\right) e^{-i \omega t} d t=\frac{i \omega}{c} \int_{0}^{l} k(z) e^{-i \frac{2 \omega}{c} z} d z$,

where $i$ is the imaginary unit, $\omega=2 \pi f$ is the angular frequency, $k(z)$ is the coupling function to the fields at point $z$, and the velocity of the beam is approximated by the speed of light $c$.

Linnecar has proposed an exponentially decaying function for the coupling function $k(z)$ as [10]

$$
k_{\text {linnecar }}(z)=k_{0} e^{-\frac{a z}{l}},
$$

where $k_{0}$ is a coupling constant, $a$ is a positive dimensionless parameter defining the degree of the exponential tapering of the electrode. For the coupling function $k_{\text {linnecar }}(z)$, the transfer function $F(\omega)$ is calculated as

$$
F(\omega)=\frac{k_{0}}{2} \frac{i 2 \omega l\left(1-e^{-a-i \frac{2 \omega l}{c}}\right)}{c\left(a+\frac{i 2 \omega l}{c}\right)} .
$$

One characteristic feature of Linnecar's exponential electrode is that the magnitude response keeps oscillating with increasing frequencies,

$$
F(\omega) \sim \frac{k_{0}}{2}\left(1-e^{-a-i \frac{2 \omega l}{c}}\right),
$$

namely, it never decays to zero.

Figure 1 shows an example of the absolute value (amplitude) of the transfer function $F(\omega)$ for the parameters

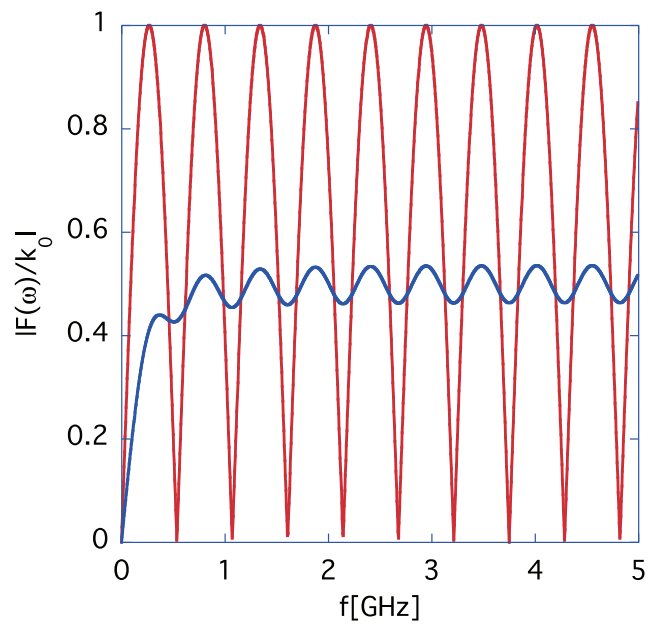

FIG. 1. The theoretical result of the transfer function $|F(\omega)|$ for Linnecar's exponential electrode for the case of $a=2.63$ and $l=280 \mathrm{~mm}$ (the blue line). The red line shows the amplitude of the transfer function for a rectangular electrode. 


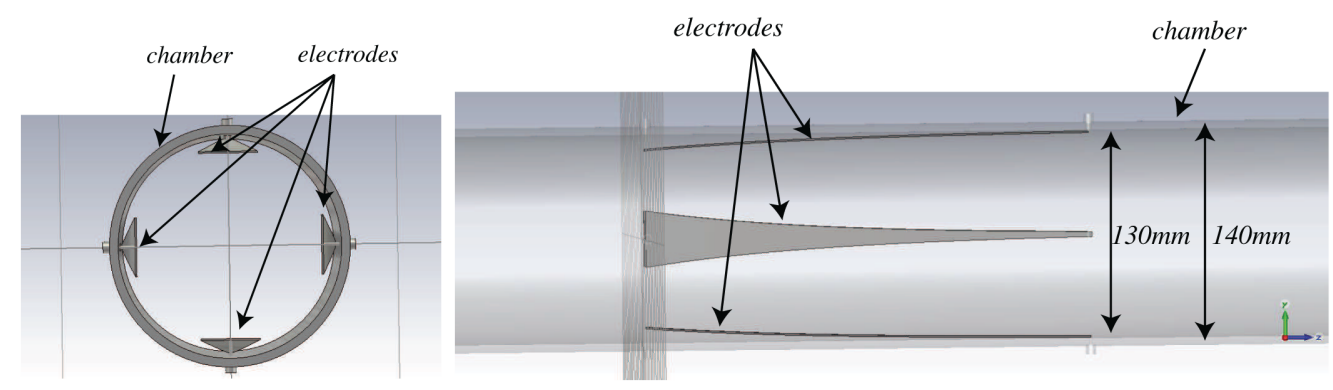

FIG. 2. The simulation model for a chamber housing the exponential electrodes. Front view (left) and side view (right).
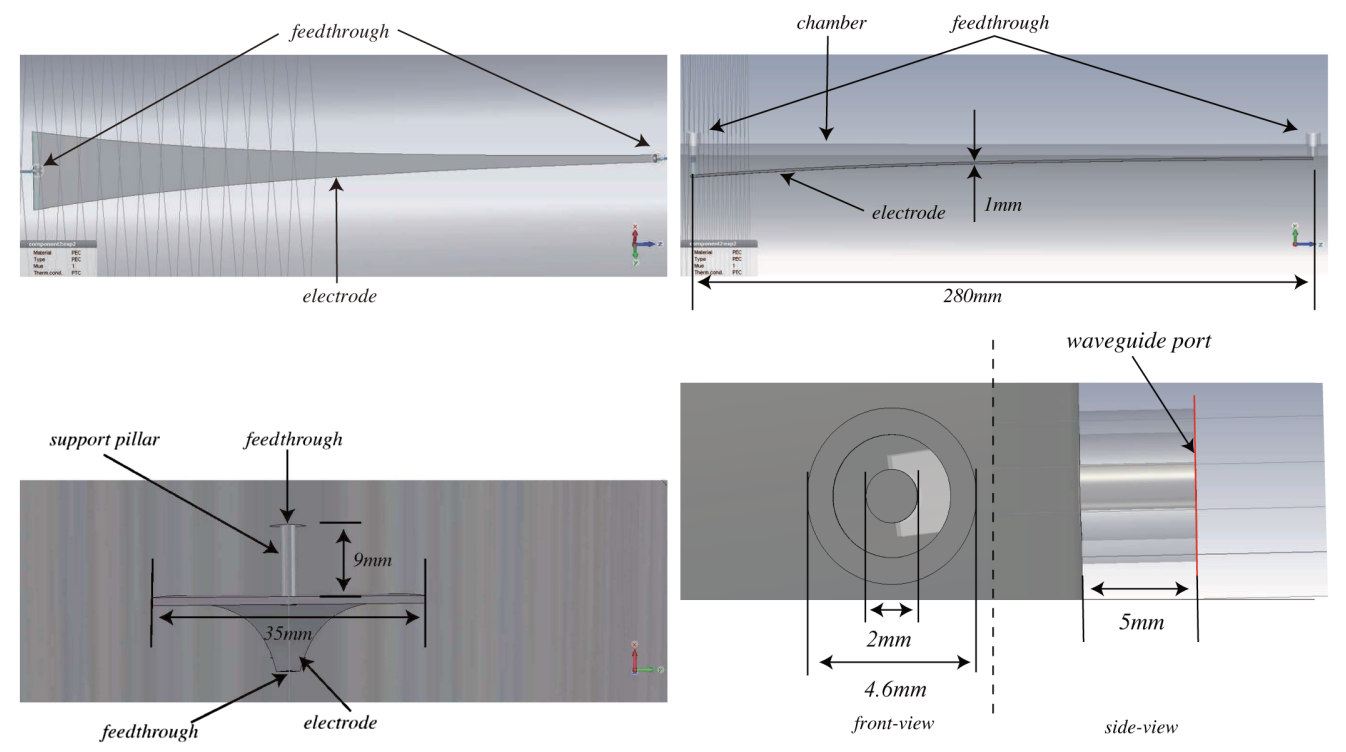

FIG. 3. The simulation model of the exponential electrode and the feedthrough. Top view (the upper left), side view (the upper right), and front view (the lower left) of the electrode. The lower-right figure shows the front and the side views of the feedthrough.

$a=2.63$ and $l=280 \mathrm{~mm}$ (the blue line) [9]. For comparison, the amplitude of the transfer function for a rectangular electrode is shown by the red line.

Three-dimensional simulations were performed by using CST PARTICLE STUDIO [13]. Figure 2 illustrates the simulation model used. The inner $\left(a_{1}\right)$ and outer radii of the chamber are 65 and $70 \mathrm{~mm}$, respectively, that are typical sizes used at MR in J-PARC [1]. Four electrodes are placed 90 degrees apart inside the chamber. Figure 3 shows the scaled figures of the electrodes and the feedthrough. The width of the electrode at the upstream end is $35 \mathrm{~mm}$. The thickness of the electrode is $1 \mathrm{~mm}$. The electrode is bent down toward the downstream proportionally to its width to keep the characteristic impedance of the electrodes to be constant $(50 \Omega)$ along the longitudinal direction. All feedthroughs are modeled by the coaxial structure with $1 \mathrm{~mm}$ inner and $2.3 \mathrm{~mm}$ outer radii. Both ends of the electrodes are terminated with four $50 \Omega$ Waveguide Ports outside the chamber behind the feedthroughs. The rms bunch length used for the wake solver module of CST Studio is $10 \mathrm{~mm}$ and the total charge is $1 \mathrm{nC}$. The wake potential is calculated for length of $0.5 \mathrm{~m}$.
The transfer function is proportional to the Fourier transform of the beam induced-voltages at the upstream port (the wider end of the electrode), divided by the Fourier transform of the beam current. Figure 4 shows the

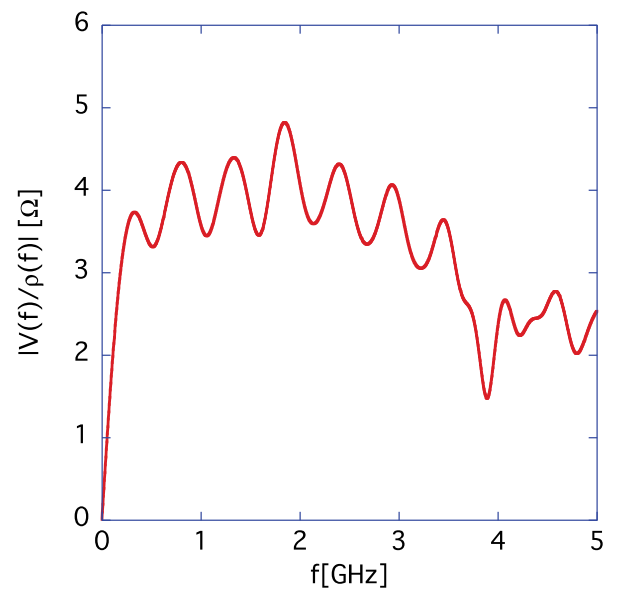

FIG. 4. The simulation result for the Linnercar's exponential electrode. 
simulation result. It roughly reproduces the theoretical transfer function shown in Fig. 1 below $2 \mathrm{GHz}$. The amount of the peak to peak oscillation amplitude is about $1 \Omega$, while the average value of the signal strength itself is about $4 \Omega$ between $0.5 \mathrm{GHz}$ and $2 \mathrm{GHz}$. Thus, the modulation amplitude is found to be about $25 \%$ below $2 \mathrm{GHz}$. It should be pointed out that this result corresponds to the ideal performance of Linnercar's exponential electrode when it is precisely fabricated and carefully set up, which is quite difficult in practice.

\section{THE TRANSFER FUNCTION OF ELECTRODES TAPERED BY LINEAR FUNCTIONS}

In this section, we investigate if a similar or even better performance can be obtained by adopting a much simpler shape for the electrode, easy to fabricate and set up. For this case, we study two linearly tapered shapes.

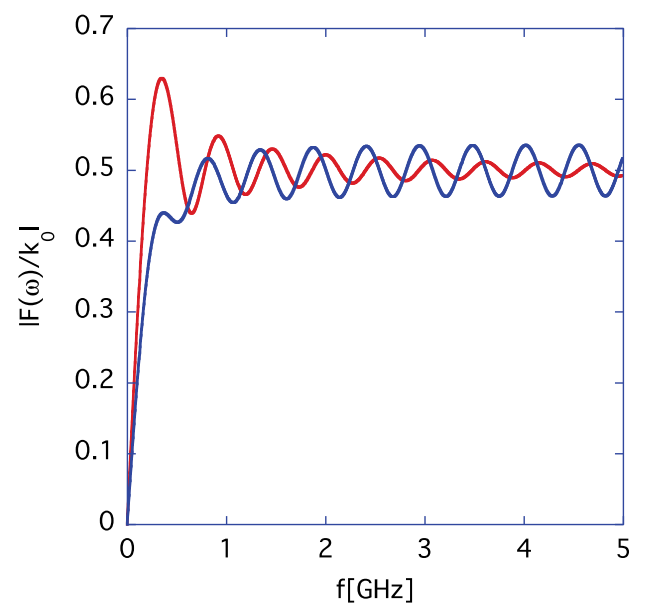

FIG. 5. The transfer function of the triangle electrode (red) and the one for the exponential electrode (blue).
The simplest shape next to the conventional rectangle is a triangle. It requires no bending to attain a good impedance matching: it is enough to place a flat triangle plate with tilt toward the downstream.

The coupling function of the triangle electrode is given by

$$
k_{\text {triangle }}(z)=k_{0} \frac{(l-z)}{l} .
$$

The transfer function is given by

$$
F(\omega)=\frac{k_{0}}{2} \frac{i\left(1-i \frac{2 \omega l}{c}-e^{-i \frac{2 \omega l}{c}}\right)}{\frac{2 \omega l}{c}} .
$$

The transfer function of the triangle electrode length $l=280 \mathrm{~mm}$ is shown in Fig. 5 by the red line. The blue line is for the exponential electrode. The transfer function of the triangle electrode damps toward high frequency, while that of the exponential electrode keeps oscillating. On the other hand, the triangle electrode has a large overshoot at low frequency.

Three-dimensional simulations are performed for the triangle electrode with the same parameters and setup as for the exponential one: the width of the wider end of the electrode is $35 \mathrm{~mm}$, and four electrodes are placed 90 degrees apart inside the chamber. The simulation model is shown in Fig. 6.

The impedance distribution along the electrode is calculated by using time domain reflectometry (TDR) method. Both the upstream (the wider end) and the downstream port of the electrode (the narrow end) are terminated with Waveguide Ports behind the feedthrough. The time domain signal at the upstream port is calculated by using the transient solver of CST MICROWAVE STUDIO [13]. The result is shown in Fig. 7. We can identify the sharp
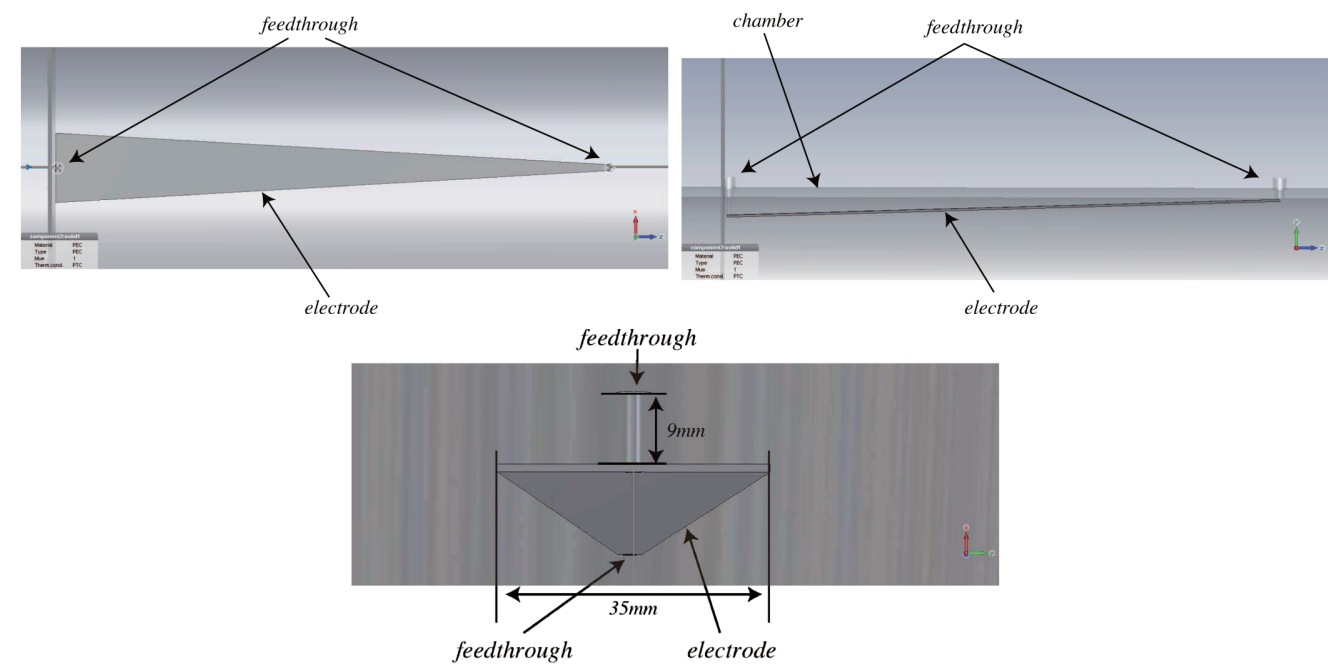

FIG. 6. The simulation model for the triangle electrode. Top view (the upper left), side view (the upper right) and front view (the lower) of the electrode. 


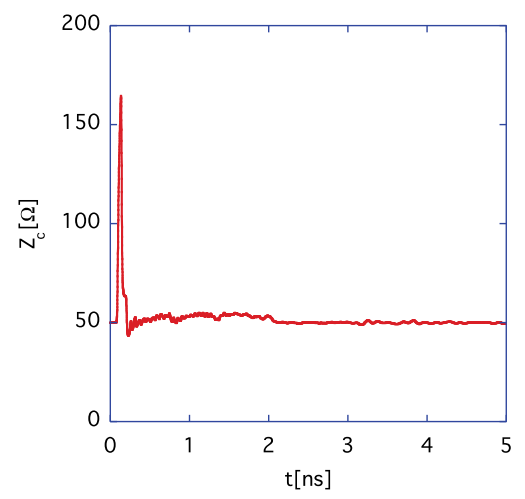

FIG. 7. The simulation result of TDR for the triangle electrode.

impedance peak at the pillar on the wider end, which is inevitable in this electrode.

Now, let us calculate the beam induced-voltages on the electrodes by using wake solver of CST PARTICLE STUDIO [13]. The left and the right figures in Fig. 8 show the injection beam profile and the beam induced-voltage

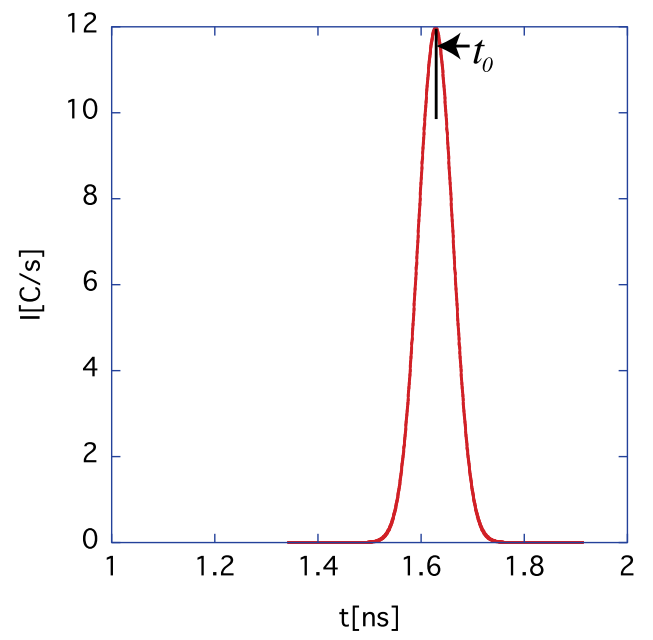

at the upstream port, respectively. The time $t_{0}$ shows the arrival time of the peak current of the beam at the upstream port, and $t_{1}$ is the corresponding time in the induced voltage.

Figure 9 shows the Fourier transform of the beaminduced voltage divided by that of the beam current. The red line in the right figure shows the phase of the result. The black line shows the line $\phi=-2 \pi f\left(t_{1}-t_{0}\right) \cdot 180 / \pi$, for reference. The simulation result almost follows the ideal linear line.

The left figure in Fig. 9 shows the amplitude of the results. The theoretical result (shown by the red line in Fig. 5) is based on the transmission line model, where only TEM modes are excited, while all modes are taken into account in the simulations. The resonance peaks are clearly identified around the transverse magnetic (TM) waveguide modes:

$$
\begin{aligned}
f_{c}= & \frac{0.114}{a_{1}}\left(\simeq 1.76 \mathrm{GHz} \text { for } a_{1}=65 \mathrm{~mm}\right), \\
& \frac{0.262}{a_{1}}\left(\simeq 4 \mathrm{GHz} \text { for } a_{1}=65 \mathrm{~mm}\right) \ldots .
\end{aligned}
$$

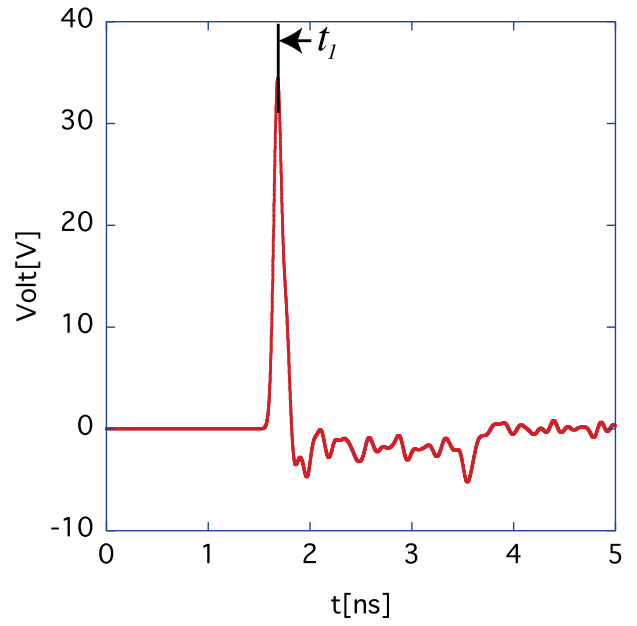

FIG. 8. The injection beam profile and the beam-induced voltage at the upstream port for the triangle electrode.
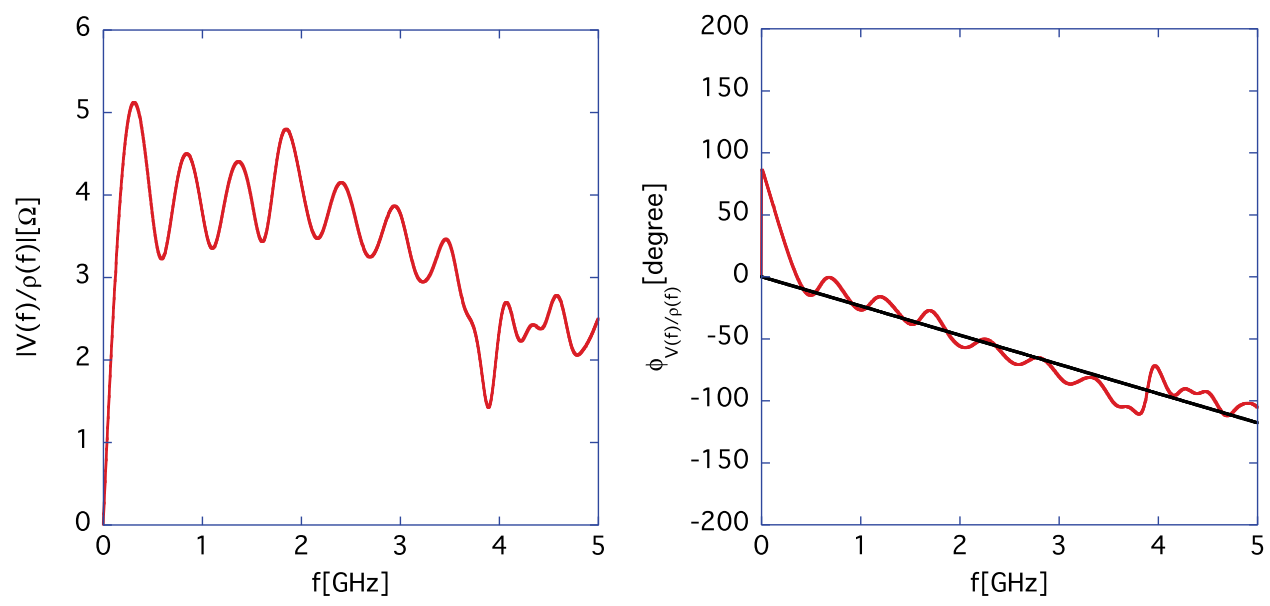

FIG. 9. Fourier transform of the beam induced-voltage divided by that of the beam current for the triangle electrode. 

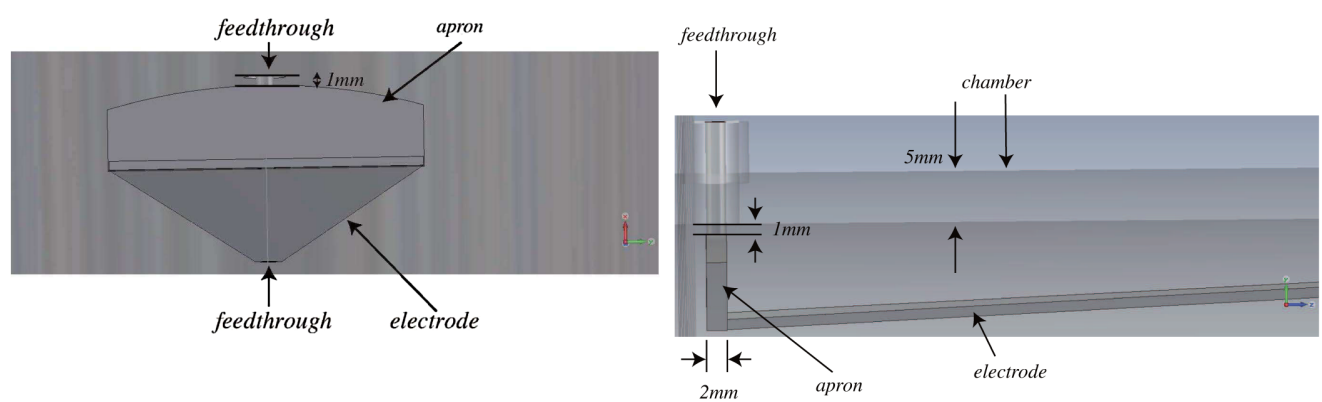

FIG. 10. The simulation model of the apron.
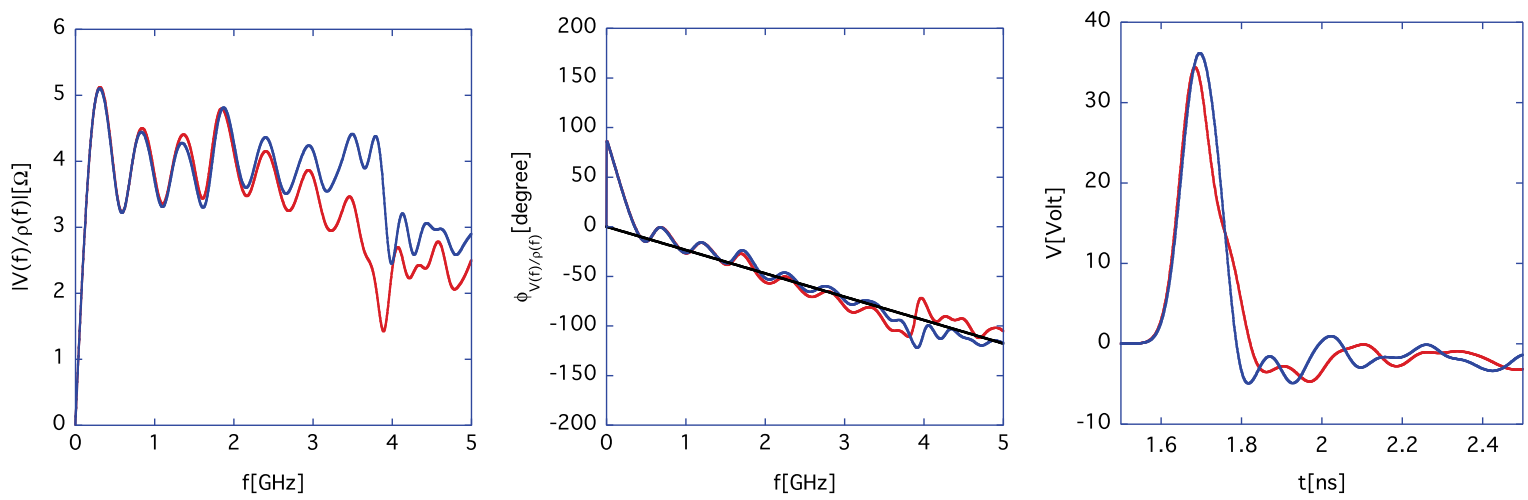

FIG. 11. The simulation results for the triangle electrode with (blue) and without (red) apron.

There is a 9 mm-high gap between chamber wall and upstream electrode (See Fig. 6.), bypassing parts of the image current to the chamber instead of inducing all the image current as the displacement current on the electrode. For short wave-length modes, this gap prevents a smooth flow of the displacement current and thus the image current on the electrode is reduced in the high frequency regime. This explains why the magnitude response declines at frequencies above the first TM-mode.

To reduce this gap for the image current, and thus to enable its smooth transition to the electrode, we attach a plate perpendicular to the upstream edge of the electrode (See
Fig. 10.). We call this plate "an apron" hereafter. With the apron, the gap between the electrode and the chamber is now reduced from $9 \mathrm{~mm}$ to $1 \mathrm{~mm}$ (Compare Fig. 6 with Fig. 10.).

Simulation results with (blue) and without (red) apron are compared in Fig. 11. The left and the center figures show the amplitude and the phase of the result, respectively. The right figure shows the beam induced-voltage. The black line shows the reference linear line as in Fig. 9. The blue line (with apron) demonstrates that the scheme is effective up to at least the second TM-mode.

The apron effect appears to be independent on the chamber size. The result for $a_{1}=40 \mathrm{~mm}$ is shown in Fig. 12,

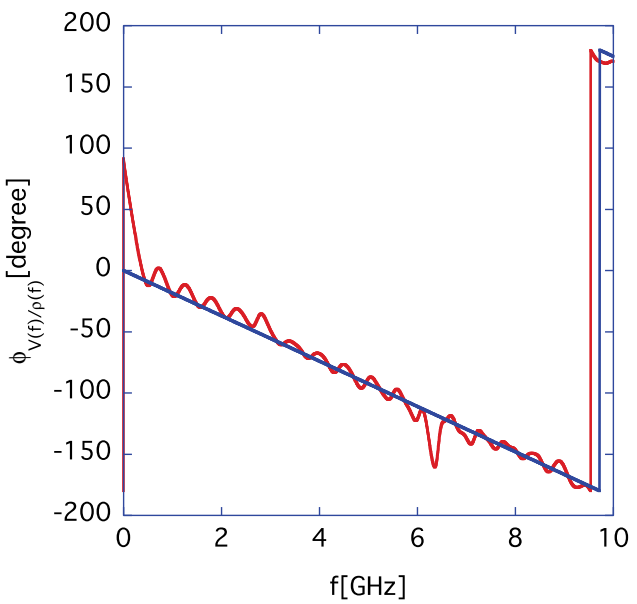

FIG. 12. The simulation results for $a_{1}=40 \mathrm{~mm}$ chamber housing the triangle electrodes with apron. 


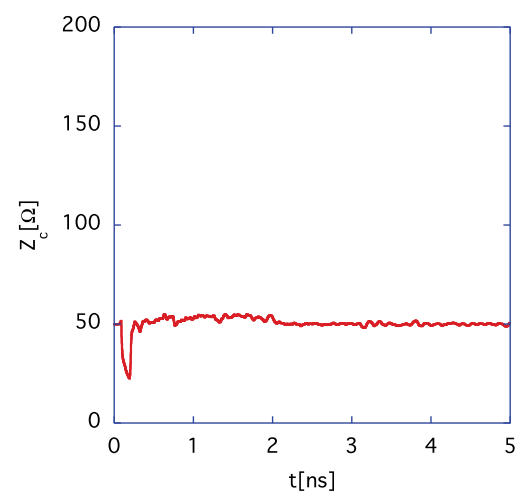

FIG. 13. The simulation result of TDR for the triangle electrode with the apron.

where the chamber houses four apron-attached electrodes with $270 \mathrm{~mm}$ long and $20.8 \mathrm{~mm}$ width at the wider end. The resonance structures move to the higher frequency following Eq. (7), and are identified around $f_{c}=2.86$ and $6.56 \mathrm{GHz}$ in this geometry. As expected, the signal strength is maintained up to the second TM-mode $(\sim 6.55 \mathrm{GHz})$ by the apron.
The impedance distribution along the electrode is simulated by using the TDR method, as well. The result is shown in Fig. 13. In comparison with Fig. 7, the impedance mismatch on the wider end is significantly reduced by the apron.

Also for the exponential electrodes, the apron improves the magnitude response at high frequencies. Figure 14 shows the frequency response of the exponential electrode without (red) and with (blue) apron: the improvement is clearly visible.

Now, let us compare the simulation results for the triangle electrode with apron (the blue line of Fig. 11) with that for the exponential one with apron (the blue line of Fig. 14). No significant difference is observable, except the overshoot at low frequency in the triangle electrode.

The next step is to mitigate this overshoot effect. This can be done by introducing one more complexity in the shape, but the final shape must be simple and easy enough for fabrication and setup.

One solution is to replace the long, straight sides of the triangle by a three-point polyline. This deformation transforms the triangle to a concave pentagon. As shown in Fig. 15, the concave pentagon electrode needs to be bent
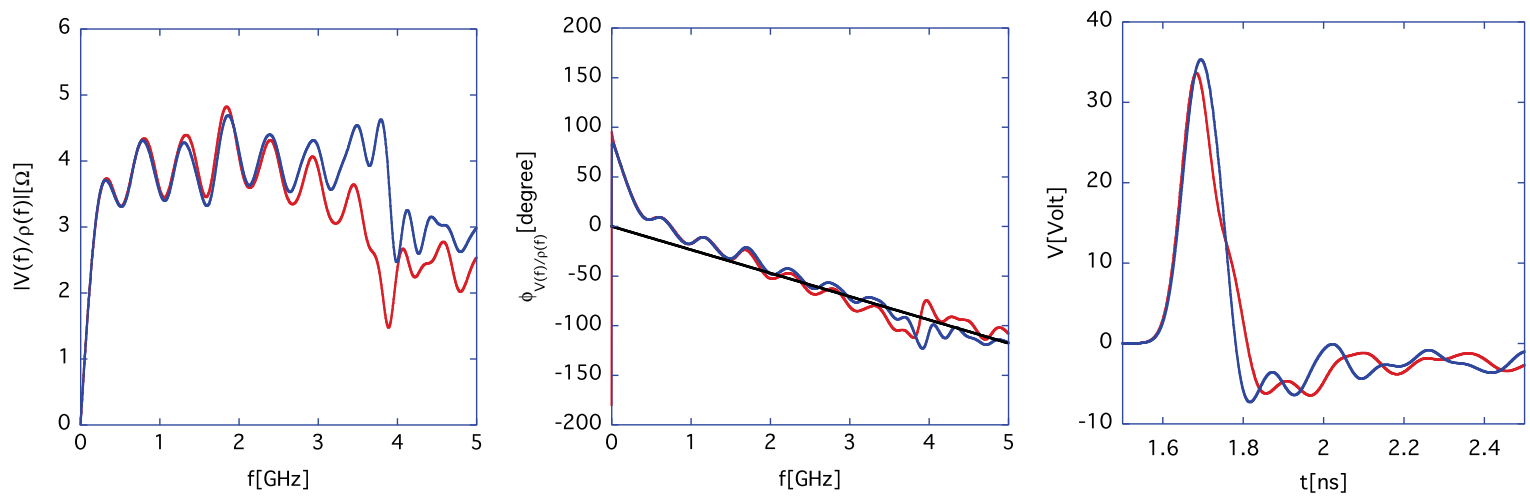

FIG. 14. The simulation result for the exponential electrode with (blue) and without (red) apron.

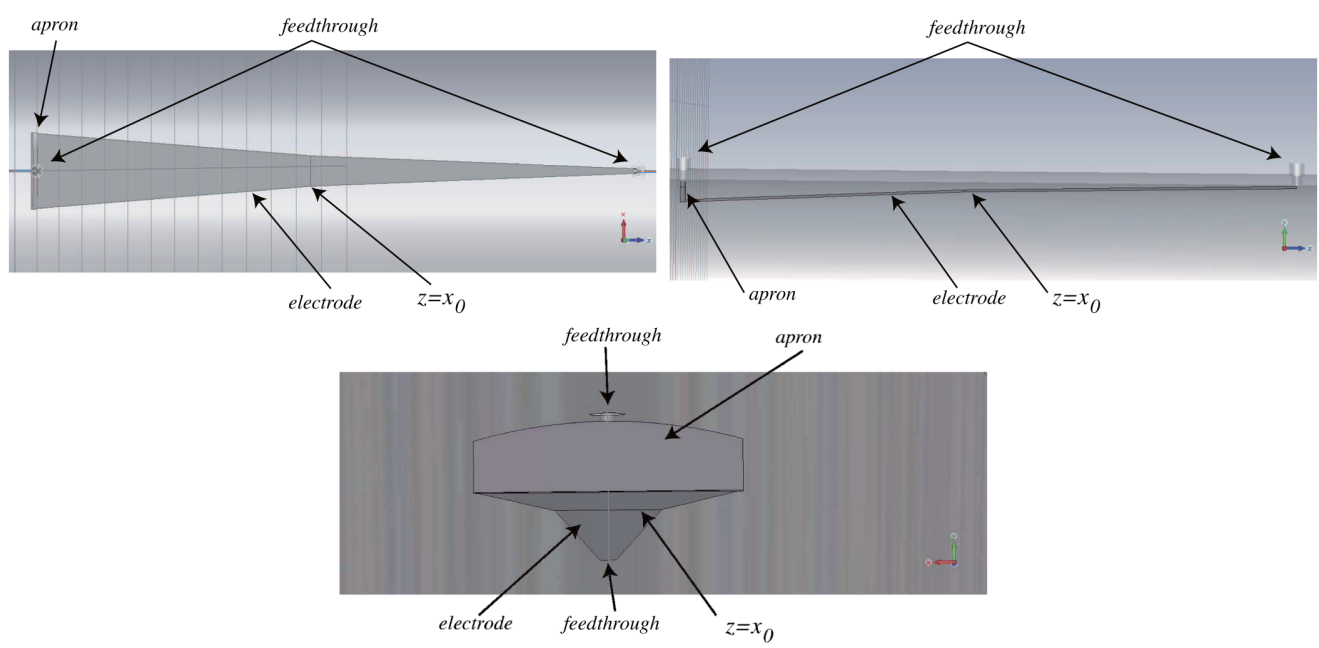

FIG. 15. Schematics of the concave pentagon electrode with a dent at $z=x_{0}$ toward the chamber. Top view (the upper left), side view (the upper right) and front view (the lower) of the electrode. 


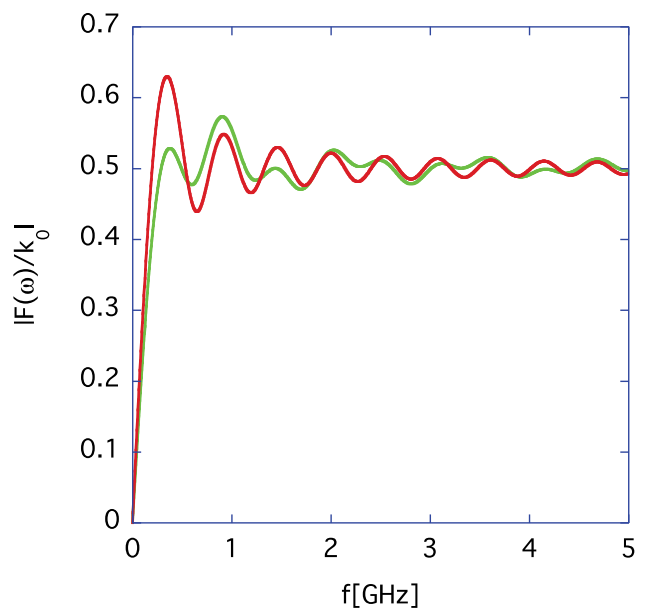

FIG. 16. The transfer function $|F(\omega)|$ for the concave pentagon electrode (green). That for the triangle electrode is shown by the red line for comparison.

only once at the middle point of the polylines for a good impedance matching. Its fabrication and setup remain easy.

The coupling function is given by

$$
k_{\text {two-linear }}(z)=\left\{\begin{array}{l}
k_{0}\left(1-\frac{\left(1-y_{0}\right) z}{x_{0}}\right), \quad \text { for } z \leq x_{0}, \\
-k_{0} \frac{y_{0}(z-l)}{\left(l-x_{0}\right)}, \quad \text { for } z>x_{0},
\end{array}\right.
$$

where $x_{0}$ and $y_{0}$ specify a middle point location. The concave pentagon electrode will be a slight deviation from the original triangle shape, with a small bend at $z=x_{0}$.

The choice of the position of the middle point provides a degree of freedom for optimization of the frequency response at low frequency. The optimal position of the middle point provides

$$
\begin{aligned}
& x_{0}=\frac{8.5}{20} l, \\
& y_{0}=\frac{7.5}{20} .
\end{aligned}
$$

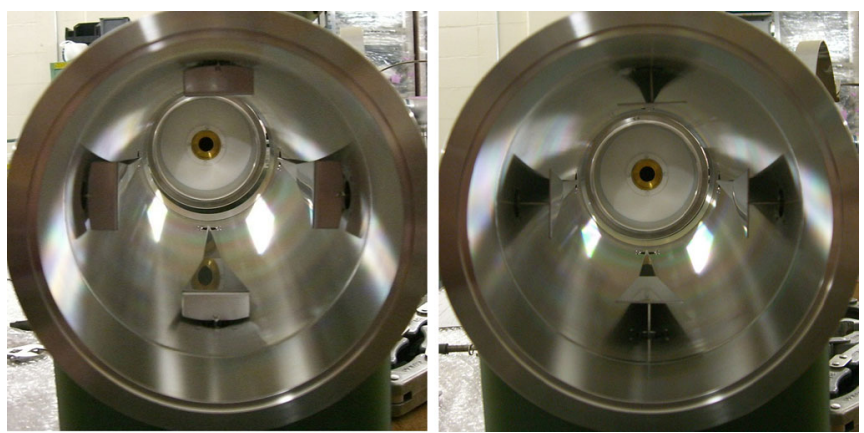

FIG. 18. Photographs of the concave pentagon electrodes with (left) and without the apron (right).

The theoretical transfer function of the concave pentagon electrode is shown by the green line in Fig. 16. For comparison, that of the triangle electrode is denoted by the red line. Simulation results with apron (blue) and without apron (red) are shown in Fig. 17. They clearly illustrate that the overshoot problem of the triangle electrode at low frequency is significantly mitigated in the concave pentagon electrode.

\section{MEASUREMENT RESULTS}

We have measured the frequency responses of the triangle and the concave pentagon electrodes with and without apron (See Fig. 18.). The schematic view of the measurement setup is shown in Fig. 19. The inner conductor is installed in the chambers with $134 \phi$, where the $306 \mathrm{~mm}$ long four electrodes are housed. The inner conductor and the chambers make a coaxial structure. One port of the network analyzer is connected to the port at the entrance of the chamber, while the other port is connected to the wider end (the upstream side) of the electrode. The chamber is sandwiched between two conical tapered coaxial section [14] to suppress reflection effects while keeping a constant $50 \Omega$ characteristic impedance for the network analyzer operation. The exit
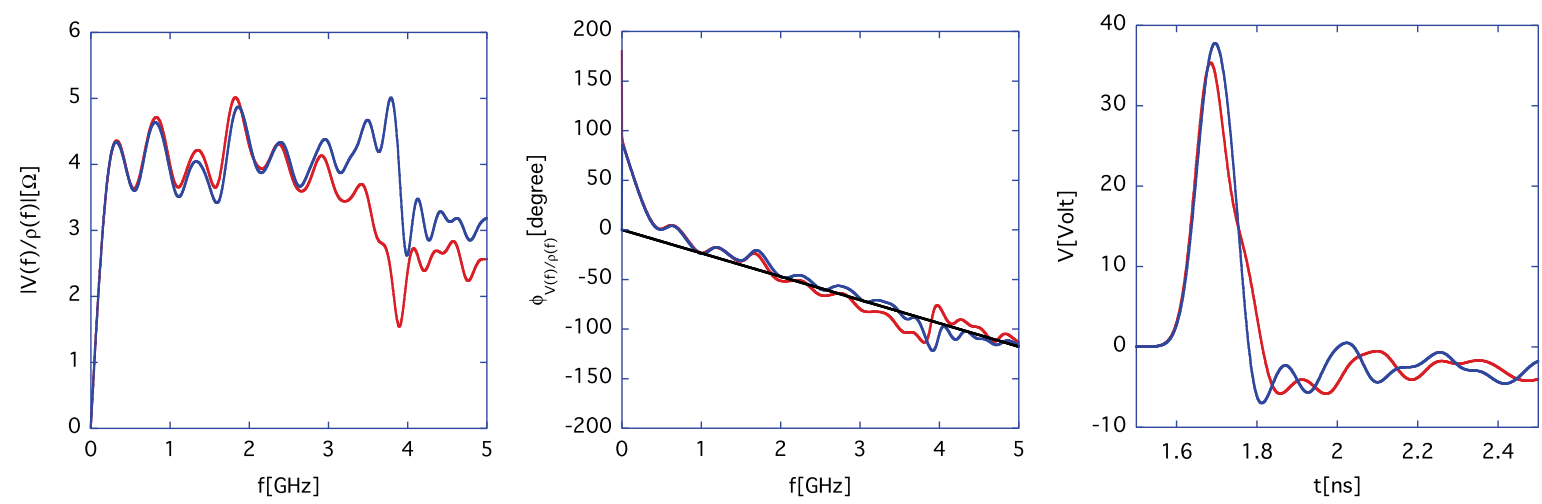

FIG. 17. The simulation result of the concave pentagon electrode. The blue and the red lines show the results with and without apron, respectively. 


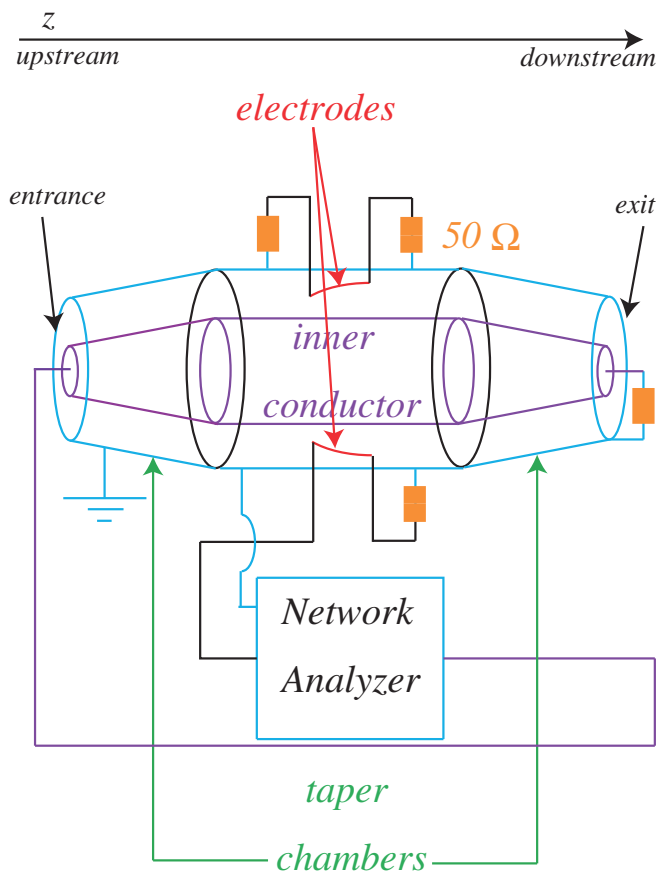

FIG. 19. A schematic of the measurement setup.

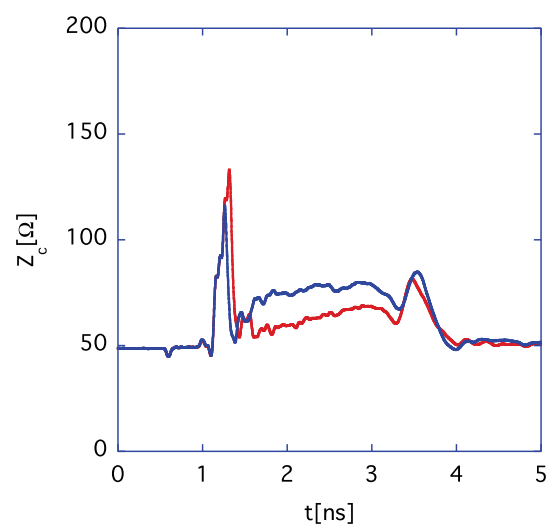

(the downstream side) of the chamber is terminated with the matched resistor.

The transfer function is estimated by measuring the transmission coefficient $S_{21}$ (See Appendix C.). The transmission coefficient $S_{21}$ is measured with 4-port Agilent Technologies ENA Series Network Analyzer E5071C [15]. The calibration is done by 2-port electric calibration module $85092 \mathrm{~b}$. The measurement is done by sweeping the frequency of the input signal (the sinusoidal function with single-frequency).

At the same time, the impedance distribution along the electrode is measured by using TDR method. The downstream port of the electrode (the narrow end) is terminated with the $50 \Omega$ resistor behind the feedthrough. The upstream port is connected to a Tektronix DSA8200 Sampling Oscilloscope through the another feedthrough [16].

Measurement results are shown in Fig. 20. The left figure of Fig. 20 shows the measured result of TDR for the electrode with (blue) and without (red) apron. The two peaks correspond to the position of the pillars on the electrode. The impedance of the electrode fluctuates

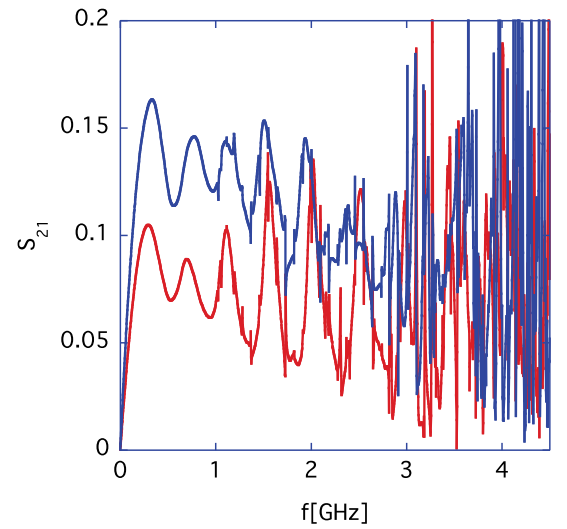

FIG. 20. The measured results of TDR (left) for the triangle electrode with (blue) and without (red) apron. The measured $S_{21}$ (right) with (blue) and without (red) apron.
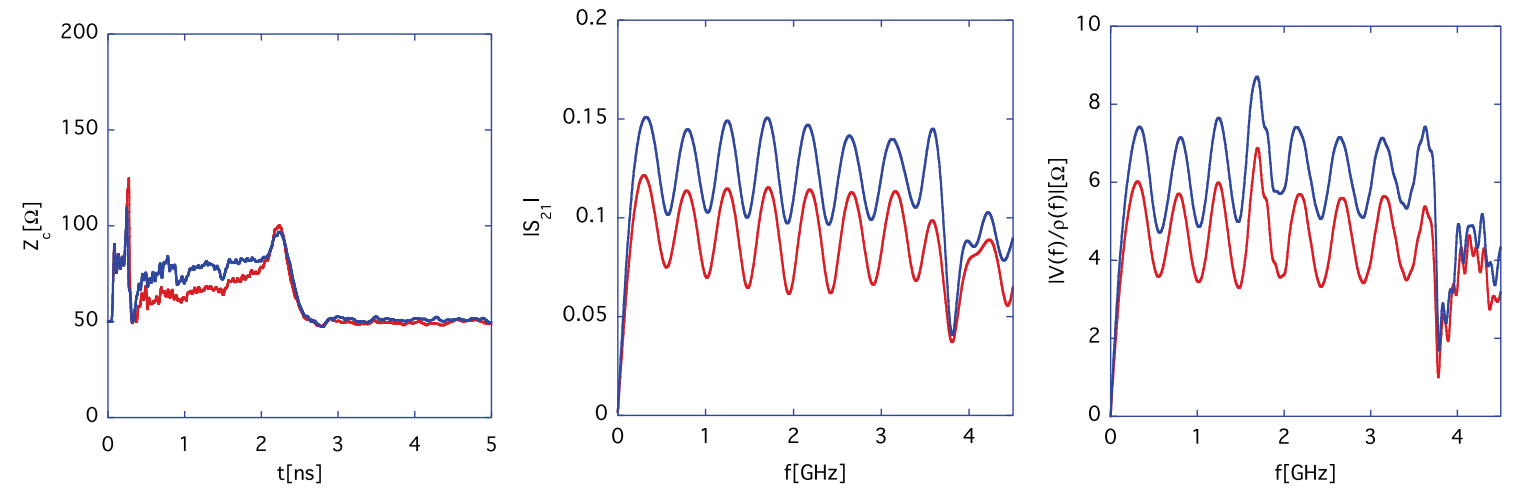

FIG. 21. The simulation results $(l=306 \mathrm{~mm}, 134 \phi)$ with artificial impedance mismatching to simulate the measurement results shown in Fig. 20: TDR (left), $S_{21}$ (middle) and the beam-induced voltage at the feedthrough divided by the beam distribution current for the triangle electrode (right) where the wake potential is calculated as a length of $3 \mathrm{~m}$. The blue and the red lines show the result with and without apron, respectively. 


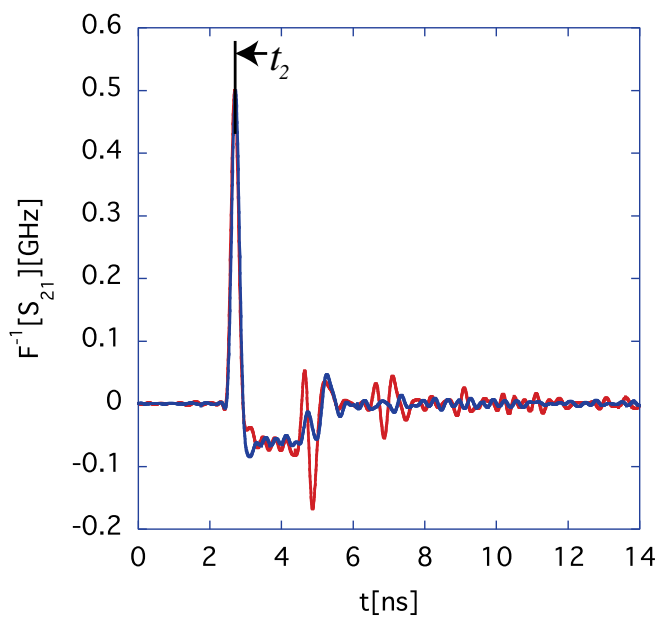

FIG. 22. Inverse Fourier transformed $S_{21}$ for the triangle electrode with (blue) and without (red) apron.
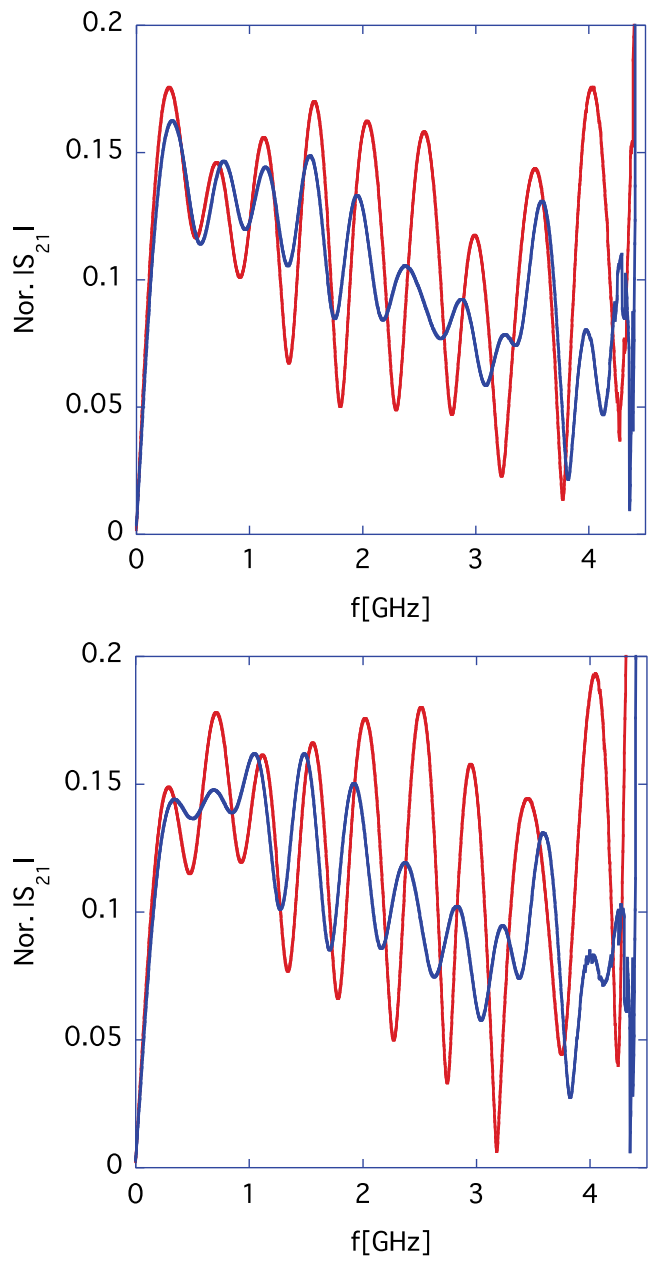

between $50 \Omega$ and $80 \Omega$. The right figure of Fig. 20 shows the measured $S_{21}$ for the triangle electrode with (blue) and without (red) apron.

Simulations with an impedance mismatch have been performed to simulate the measurement results. The left figure of Fig. 21 is the characteristic impedance of the electrode used in the simulations. The comparison of the results with those in Figs. 7 and 13 illustrates how large the impedance mismatch along the electrode is. Only the mismatch at the pillar on the upstream port is somewhat mitigated, comparing the result in Fig. 7.

The middle and the right figures of Fig. 21 show $S_{21}$ and the frequency response (the beam induced voltage at the feedthrough divided by the beam distribution current), respectively. The blue and the red lines show the result with and without apron, respectively. We see that the middle figure $\left(S_{21}\right)$ fairly reproduces the behavior of the right figure (The inner conductor is replaced by a beam.). Thus, we can conclude that the characteristic of the transfer function can be investigated by measuring the parameter $S_{21}$.
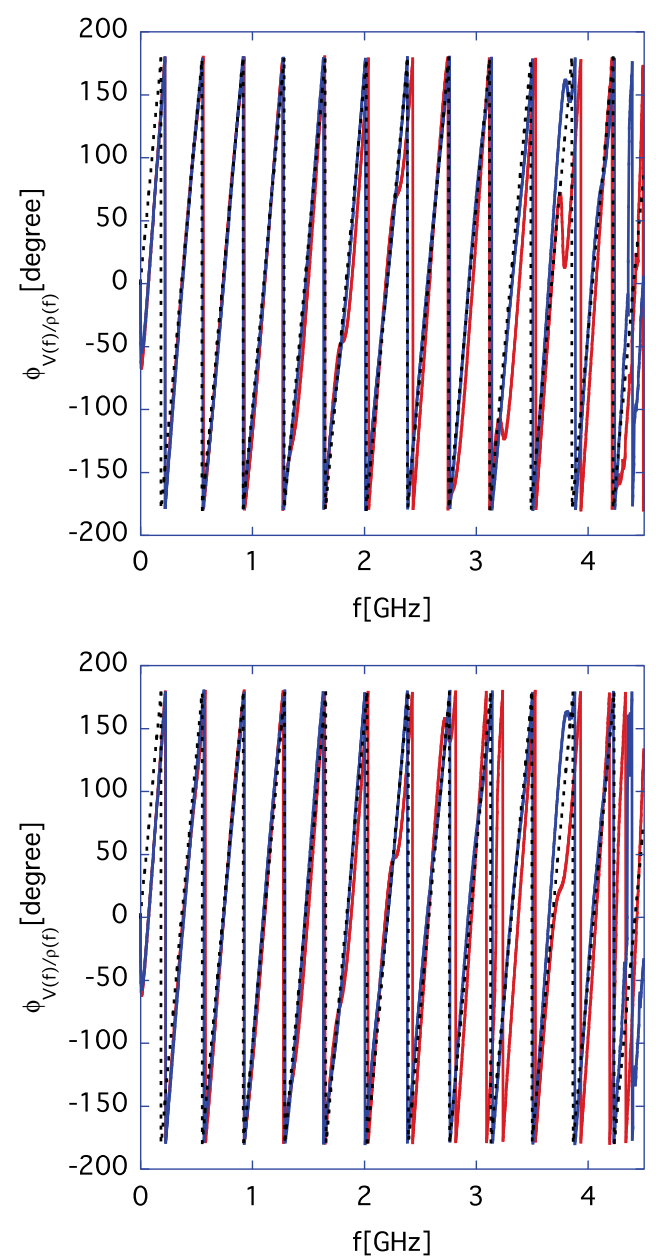

FIG. 23. Measured $S_{21}$ after the TDG treatment for the triangle (top) and the concave pentagon (bottom) electrodes with (blue) and without (red) apron. The dotted line shows the line $\phi=2 \pi f t_{2} \cdot 180 / \pi$ for reference, where $t_{2}$ is the time at the peak point of inverse Fourier transform of $S_{21}$. 
The different signal strength with and without apron is due to a setup error, where the electrode with apron was placed closer to the chamber center than that without apron. As a result, the electrode with apron has a stronger coupling to the beam, and thus a larger impedance. For the following discussion of the results, the difference in signal strength will be adjusted so that the impedances of both configurations are better matched.

Now, let us examine the inverse transform of the measured $S_{21}$ in time-domain. The limited span [0-4.5 GHz] of the network analyzer produces the ripples in the straightforward inverse transform. In order to avoid them, let us define the inverse transform of $S_{21}$ as

$$
F^{-1}\left[S_{21}\right]=2 \Re\left[\int_{0}^{4.5 \times 10^{9}} d f e^{i 2 \pi f t} S_{21}\right] W(f),
$$

by making use of Hahn Window function [17], i.e.,

$$
W(f)=0.5-0.5 \cos \left[\frac{2 \pi\left(f-4.5 \times 10^{9}\right)}{9 \times 10^{9}}\right]
$$

The results are shown in Fig. 22. The blue and the red traces show the results with and without apron, respectively. The red trace (without apron) shows that a substantial reflection signal is created by a mismatch between the characteristic impedance of the electrode and the terminal impedance $(50 \Omega)$ of the feedthrough. On the other hand, this effect is quite satisfactory suppressed for the electrode with apron (the blue trace). It means that the spatial impedance produced by the apron partially compensates the difference between the characteristic impedance of the electrode and the terminal impedance of the feedthrough.

As an attempt to cut off multiple reflection effects, we apply the time domain gating (TDG) for the period between 2 and $6 \mathrm{~ns}$ on the measured $S_{21}$. The results for the triangle electrode are shown in the top figures of Fig. 23 with (blue) and without (red) apron, respectively. We can clearly see that the apron is quite effective to reduce the fluctuation of the frequency response. The excitation of the second TM-mode $(\sim 3.7 \mathrm{GHz})$ can be identified in the blue line. The gradual decline of the signal strength up to the second TM-mode can be mitigated by further improving the impedance matching to $50 \Omega$.

The mismatches in the feedthrough elements at each end of the electrodes are significant contributors to the frequency response variations and "ringing" at high frequency in practice. In our measurement, $N$-type connectors were adopted as the feedthroughs. The replacement of them by SMA (SubMiniature version A) connectors can produce a better frequency characteristic.

We applied the same treatment on the measured $S_{21}$ signals for the concave pentagon electrode. The bottom figures of Fig. 23 show the results. Their behaviors are similar to those for the triangle electrode, except at low frequency where the overshoot of the signal is significantly reduced. As a result, the overall frequency response becomes flatter than the triangle electrode up to about $4 \mathrm{GHz}$.

\section{CONCLUSIONS}

We have built and tested a Linnercar-type exponential electrode for J-PARC SBPM [2]. Since it was difficult to bend it exponentially as required for a good impedance matching, we kept it straight as a flat plate and installed it as part of the SBPM [11]. Figure 4 shows the simulation results for the ideal case with precise fabrication and setup. In real measurements, however, we have never obtained such a good frequency performance. The measured frequency response after the TDG treatment is shown in Fig. 24 (In this measurement, the chamber with the exponential electrodes was capped with straight ducts, not tapers, from the both sides $[2,11]$. Thus, the measurement becomes inaccurate at high frequency.). It is far from the theoretical and simulation predictions.

Then, we started to suspect that once we replace the conventional rectangle electrode by a triangle or its alike, a further increase of the complexity of the shape will hardly improve the frequency response. In fact, the frequency response of the ideal exponential electrode (Fig. 14) is surprisingly similar to that for the concave pentagon (Fig. 17). The concave pentagon is much easier to fabricate and setup, since a concave-pentagon shaped flat plate needs

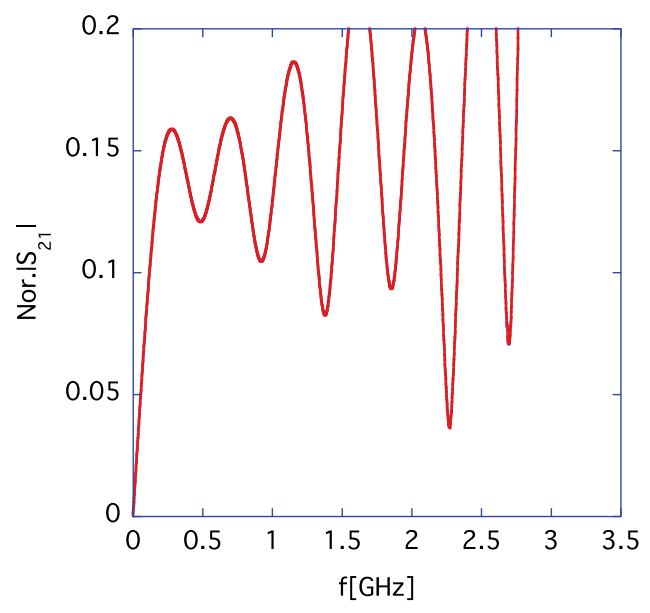

FIG. 24. Measured $S_{21}$ after the TDG treatment for the exponential electrodes without apron. 
to be slightly bent only once at the middle concave point. Nevertheless, it can produce the frequency response as good as one for the ideal exponential electrode.

The simulation results demonstrate that the apron attached on the wider end of the electrode helps to maintain the high signal strength up to the second TM-mode (about $4 \mathrm{GHz}$ for a $65 \mathrm{~mm}$ radius chamber). We expect that the gradual decline of the frequency response at high frequency in measurements can be mitigated by improving the impedance matching in the fabrication process.

\section{ACKNOWLEDGMENTS}

The authors would like to thank M. Tobiyama for discussions. The authors also would like to thank all members of J-PARC project at JAEA/KEK. This work was partially supported by MEXT KAKENHI Grant No. 25105002, Grant-in-Aid for Scientific Research on Innovative Areas titled "Unification and Development of the Neutrino Science Frontier".

\section{APPENDIX A: COUPLING IMPEDANCE OF LINNECAR'S ELECTRODE}

As written in the Introduction, the slotted-coaxial kicker shows a broadband impedance. For comparison, let us calculate the beam coupling impedance of Linnecar's electrode. The simulation setup is shown in Fig. 2. The results are shown in Fig. 25. The left and the right figures show the longitudinal impedance and the wake function, respectively. The red and the blue lines in the left figure denote the real and the imaginary parts of the impedance, respectively. The impedance roughly behaves like purely inductive, and correspondingly, the wake function is close to the derivative of the $\delta$-function.

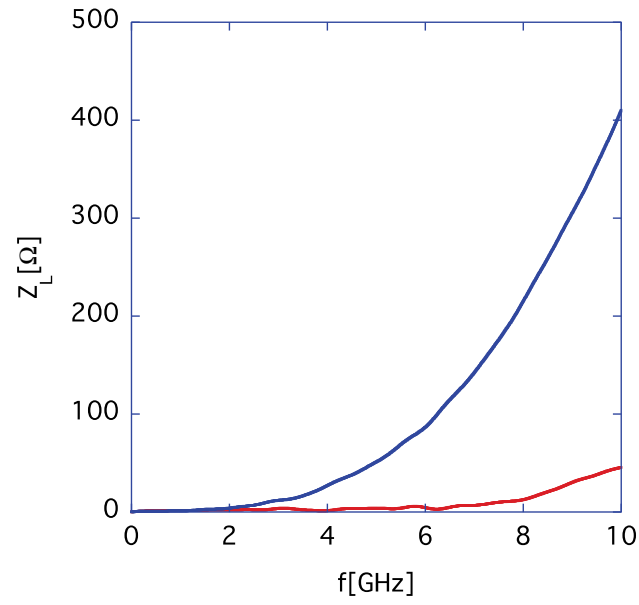

\section{APPENDIX B: RELATIONSHIP BETWEEN THE BEAM-INDUCED VOLTAGE ON ONE ELECTRODE AND THE TRANSFER FUNCTION $\boldsymbol{F}(\boldsymbol{\omega})$}

Let us imagine an electrode with the length $l$, the inductance $L$ and the capacitance $C$ per unit length in a vacuum chamber. Both ends of the electrode are terminated with resistors $R$. Based on the transmission line model, the voltage $\tilde{V}$ and the current $\tilde{I}$ at the position $z$ of the electrode, that are excited by the beam current $I_{0}$ with its frequency $f$, satisfy the following equations:

$$
\begin{gathered}
\frac{d \tilde{V}(z)}{d z}=-i \omega L(z) \tilde{I}(z)-i \omega M(z) I_{0} e^{-i \frac{\omega}{\tilde{c}} \bar{z}} \\
\frac{d \tilde{I}(z)}{d z}=-i \omega C(z) \tilde{V}(z)
\end{gathered}
$$

where $\omega=2 \pi f$ and $M(z)$ is the mutual inductance between the beam and the electrode at $z$. If the characteristic impedance $\sqrt{L / C}$ does not depend on $z$ and it is equal to $R$, the induced voltage at $z=0$ is given by

$$
\tilde{V}(z=0)=\frac{i \omega I_{0}}{2} \int_{0}^{l} d z^{\prime} M\left(z^{\prime}\right) e^{-i 2 \frac{\omega}{c} z^{\prime}}
$$

which is basically identical to the transfer function $F(\omega)$ in Eq. (1).

Following Oliver [12], the sign of the imaginary unit $i$ is chosen so that the corresponding function $v(t)$ in timedomain is given by

$$
v(t)=\int_{-\infty}^{\infty} \frac{d \omega}{2 \pi} \tilde{V} e^{i \omega t}
$$

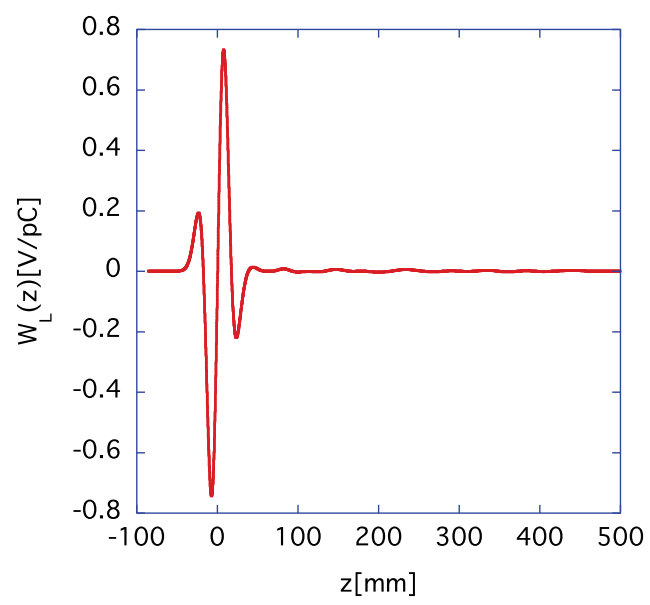

FIG. 25. The longitudinal impedance and the wake function for Linnecar's electrode. 


\section{APPENDIX C: VALIDITY OF THE MEASUREMENT SETUP}

The transfer function $F(\omega)$ can be obtained from a measured $S$-parameter $S_{21}$ in the setup shown in Fig. 19. Since a beam is replaced by the inner conductor in this setup, we need some considerations about the validity of the scheme. In this appendix, let us examine the relationship between the transfer function $F(\omega)$ and the $S$-parameter.

First, let us consider the transmission line model illustrated in Fig. 26. Port 1 and 2 are located at the entrance of the chamber and at the feedthrough on the upstream port (wide side) of the electrode, respectively. For simplicity, let us assume that they are located at the same longitudinal position at $z=0$.

Equations are given by

$$
\begin{gathered}
\frac{d V_{1}}{d z}=-i \omega L(z) I_{1}-i \omega M(z) I_{\mathrm{inner}} \\
\frac{d I_{1}}{d z}=-i \omega \frac{L_{\mathrm{inner}}}{c^{2}\left[L(z) L_{\mathrm{inner}}-M(z)^{2}\right]} V_{1} \\
+i \omega \frac{M(z)}{c^{2}\left[L(z) L_{\mathrm{inner}}-M^{2}(z)\right]} V_{\mathrm{inner}} \\
\frac{d V_{\mathrm{inner}}}{d z}=-i \omega L_{\mathrm{inner}} I_{\mathrm{inner}}-i \omega M(z) I_{1} \\
\frac{d I_{\mathrm{inner}}}{d z}=i \omega \frac{M(z)}{c^{2}\left[L(z) L_{\mathrm{inner}}-M(z)^{2}\right]} V_{1} \\
-i \omega \frac{L(z)}{c^{2}\left[L(z) L_{\mathrm{inner}}-M(z)^{2}\right]} V_{\mathrm{inner}}
\end{gathered}
$$

where $L(z), V_{1}$, and $I_{1}$ are the self-inductance, the voltage, and the current of the electrode, respectively, $L_{\text {inner }}, V_{\text {inner }}$,

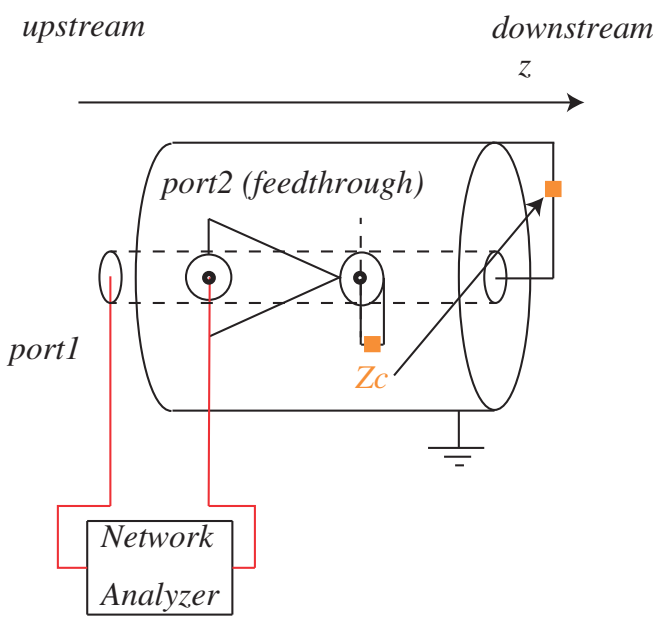

FIG. 26. A simplified schematic of the measurement setup for the transmission line model. and $I_{\text {inner }}$ are the self-inductance, the voltage, and the current of the inner conductor, respectively, and $M(z)$ is the mutual inductance between the inner conductor and the electrode.

The characteristic impedance $Z_{c}$ between the electrode and the chamber without the inner conductor is constant along $z$, while the characteristic impedance between the inner conductor and the chamber is $Z_{c}$ without the electrode.

It is quite difficult to solve Eqs. (C1)-(C4) exactly. Let us make the following two approximations to solve them: (1) that the induced voltage and the current on the inner conductor are not affected by the electrode, (2) that the coupling between the inner conductor and the electrode is weak $\left(M^{2} \ll L L_{\text {inner }}\right)$. Consequently, Eqs. (C1)-(C4) can be simplified as

$$
\begin{gathered}
\frac{d V_{1}}{d z}=-i \frac{\omega}{c} Z_{c} I_{1}-i \omega M(z) I_{\mathrm{inner}}, \\
\frac{d I_{1}}{d z}=-i \frac{\omega}{c} \frac{1}{Z_{c}} V_{1}+i \omega \frac{M(z)}{Z_{c}^{2}} V_{\mathrm{inner}}, \\
\frac{d V_{\text {inner }}}{d z}=-i \frac{\omega}{c} Z_{c} I_{\text {inner }}, \\
\frac{d I_{\text {inner }}}{d z}=-i \omega \frac{1}{c Z_{c}} V_{\text {inner }} .
\end{gathered}
$$

Solutions of Eqs. (C7) and (C8) are expressed as

$$
\begin{gathered}
V_{\text {inner }}=Z_{c}\left(A e^{-i \frac{\underline{\omega}}{c} z}-B e^{i \frac{\omega}{c} z}\right), \\
I_{\text {inner }}=A e^{-i \frac{\underline{\omega}}{c} z}+B e^{i \frac{\omega}{c} z}
\end{gathered}
$$

where $A$ and $B$ are arbitrary constants.

From the boundary condition that the port- 1 is located at $z=0$,

$$
\begin{aligned}
& A-B=\frac{\left(a_{1}^{+}+a_{1}^{-}\right)}{\sqrt{Z_{c}}}, \\
& A+B=\frac{\left(a_{1}^{+}-a_{1}^{-}\right)}{\sqrt{Z_{c}}},
\end{aligned}
$$

which is equivalent to

$$
\begin{gathered}
A=\frac{a_{1}^{+}}{\sqrt{Z_{c}}}, \\
B=-\frac{a_{1}^{-}}{\sqrt{Z_{c}}} .
\end{gathered}
$$

The other boundary condition that the inner conductor is terminated with a resistor $Z_{c}$ at the end of the chamber $z=l_{c}$ gives

$$
V_{\text {inner }}\left(z=l_{c}\right)=Z_{c} I_{\text {inner }}\left(z=l_{c}\right),
$$


which leads the relation:

$$
B=a_{1}^{-}=0 .
$$

Thus, the voltage and the current of the inner conductor are given by

$$
\begin{gathered}
V_{\text {inner }}=\sqrt{Z_{c}} a_{1}^{+} e^{-i \frac{\omega}{c} z}, \\
I_{\text {inner }}=\frac{a_{1}^{+}}{\sqrt{Z_{c}}} e^{-i \frac{\omega}{c} z} .
\end{gathered}
$$

Substituting Eqs. (C17) and (C18) into (C5) and (C6), we obtain

$$
\begin{array}{r}
\frac{d V_{1}}{d z}=-i \frac{\omega}{c} Z_{c} I_{1}-i \omega M(z) a_{1}^{+} \frac{e^{-i \frac{\omega}{c} z}}{\sqrt{Z_{c}}}, \\
\frac{d I_{1}}{d z}=-\frac{i \omega}{c Z_{c}} V_{1}+i \omega \frac{M(z)}{Z_{c}^{2}} a_{1}^{+} \sqrt{Z_{c}} e^{-i \frac{\omega}{c} z} .
\end{array}
$$

The solutions are expressed as

$$
\begin{aligned}
& V_{1}=Z_{c} A_{1} e^{-i \frac{\omega}{c} z}-Z_{c} e^{i \frac{\omega}{c} z} \int_{0}^{z} d z^{\prime} i \omega \frac{M\left(z^{\prime}\right)}{Z_{c}^{2}} a_{1}^{+} \sqrt{Z_{c}} e^{-i 2 \frac{\omega}{c} z^{\prime}} \\
& -Z_{c} B_{1} e^{i \frac{\omega}{c} z}, \\
& I_{1}=A_{1} e^{-i \frac{\omega}{c} z}+e^{i \frac{\dot{\omega}}{c} z} \int_{0}^{z} d z^{\prime} i \omega \frac{M\left(z^{\prime}\right)}{Z_{c}^{2}} a_{1}^{+} \sqrt{Z_{c}} e^{-i 2 \frac{\omega}{c} z^{\prime}}+B_{1} e^{i \frac{\omega}{c} z},
\end{aligned}
$$

where $A_{1}$ and $B_{1}$ are the arbitrary constants.

Since the port- 2 is also located at $z=0$, the constants $A_{1}$ and $B_{1}$ can be expressed using $a_{2}^{+}$and $a_{2}^{-}$as

$$
\begin{gathered}
V_{1}(z=0)=\left(a_{2}^{+}+a_{2}^{-}\right) \sqrt{Z_{c}}, \\
I_{1}(z=0)=\frac{\left(a_{2}^{+}-a_{2}^{-}\right)}{\sqrt{Z_{c}}} .
\end{gathered}
$$

Then, we obtain

$$
\begin{aligned}
& V_{1}=Z_{c} \frac{a_{2}^{+}}{\sqrt{Z_{c}}} e^{-i \frac{\underline{\omega}}{c} z}-Z_{c} e^{i \frac{i_{c}}{c} z} \int_{0}^{z} d z^{\prime} i \omega \frac{M\left(z^{\prime}\right)}{Z_{c}^{2}} a_{1}^{+} \sqrt{Z_{c}} e^{-i 2 \frac{\underline{\omega}}{c} z^{\prime}} \\
& +Z_{c} \frac{a_{2}^{-}}{\sqrt{Z_{c}}} e^{i \frac{\omega}{c} z}, \\
& I_{1}=\frac{a_{2}^{+}}{\sqrt{Z_{c}}} e^{-i \frac{\omega}{c} z}+e^{i \frac{\omega}{c} z} \int_{0}^{z} d z^{\prime} i \omega \frac{M\left(z^{\prime}\right)}{Z_{c}^{2}} a_{1}^{+} \sqrt{Z_{c}} e^{-i 2 \frac{\omega}{c} z^{\prime}} \\
& -\frac{a_{2}^{-}}{\sqrt{Z_{c}}} e^{i \frac{\omega}{c} z} .
\end{aligned}
$$

When the electrode is terminated with the resistor $Z_{c}$ at the end of the electrode $z=l$, the boundary condition:

$$
Z_{c} I_{1}(z=l)=V_{1}(z=l)
$$

provides the $S$-matrix as

$$
S_{21}=\int_{0}^{l} d z^{\prime} i \omega \frac{M\left(z^{\prime}\right)}{Z_{c}} e^{-i 2 \frac{\omega}{c} z^{\prime}},
$$

which is basically identical to the transfer function $F(\omega)$.

[1] http://j-parc.jp/index-e.html.

[2] K. Nakamura, T. Toyama, M. Okada, M. Tobiyama, Y. H. Chin, T. Obina, T. Koseki, and Y. Shobuda, Intra-bunch feedback system for the J-PARC main ring, in Proceedings of IPAC14, Dresden, Germany (2014), THOAA03, http://accelconf.web.cern.ch/AccelConf/IPAC2014/papers/ thoaa03.pdf.

[3] Y. H. Chin, T. Obina, M. Okada, M. Tobiyama, T. Toyama, K. Nakamura, and Y. Shobuda, Performance of transverse intra-bunch feedback system at J-PAC MR, in Proceedings of HB14, THO3AB01 (2014), East-Lansing, MI, http://accelconf.web.cern.ch/AccelConf/HB2014/papers/ tho3ab01.pdf.

[4] E. Mahner, T. Kroyer, and F. Caspers, Electron cloud detection and characterization in the CERN Proton Synchrotron, Phys. Rev. Accel. Beams 11, 094401 (2008).

[5] L. Faltin, Slot-type pick-up and kicker for stochastic beam cooling, Nucl. Instrum. Methods 148, 449 (1978).

[6] F. Caspers, Planar slotline pick-ups and kickers for stochastic cooling, Report No. CERN-PS-85-48-AA, 1986.

[7] C. Peschke, F. Nolden, and M. Balk, Planar pick-up electrodes for stochastic cooling, Nucl. Instrum. Methods Phys. Res., Sect. A 532, 459 (2004).

[8] J. M. Cesaratto, J. D. Fox, C. H. Rivetta, D. Alesini, A. Drago, A. Gallo, F. Marcellini, M. Zobov, S. De Santis, Z. Paret, A. Ratti, H. Qian, H. Bartosik, W. Hofle, and C. Zannini, SPS wideband transverse feedback kicker: design report, Report No. CERN-ACC-NOTE-2013-0047, SLAC report number: SLAC-R-1037, 2013.

[9] Y. Shobuda and Y.H. Chin, Optimization of electrode shape for stripline beam position monitors, Phys. Rev. Accel. Beams 17, 092801 (2014).

[10] T.P. R. Linnecar, The high frequency longitudinal and transverse pick-ups used in the SPS, CERN Report No. CERN-SPS-ARF-SPS/78/17, 1979; T. P. R. Linnecar, IEEE Transactions on NS-26 26, 3409 (1979).

[11] K. G. Nakamura, T. Toyama, M. Okada, M. Tobiyama, Y. H. Chin, T. Obina, H. Kuboki, T. Koseki, and Y. Shobuda, Fabrication of tapered coupler for intra-bunch feedback system in J-PARC main ring, in Proceedings of the 11th Annual Meeting of Particle Accelerator Society of Japan, SAOM03 (2014), Aomori, Japan (in Japanese), http://www.pasj.jp/.

[12] B. M. Oliver, Directional electromagnetic couplers, Proc. IRE 42, 1686 (1954).

[13] CST STUDIO SUITE, https://www.cst.com/. 
[14] K. G. Nakamura, T. Toyama, M. Okada, M. Tobiyama, Y. H. Chin, T. Obina, T. Koseki, and Y. Shobuda, Development of wideband BPM for precise measurement of internal bunch motion, in Proceedings of IPAC15, Richmond, VA (2015), MOPTY001, http://accelconf.web.cern.ch/ AccelConf/IPAC2015/papers/mopty001.pdf.
[15] Keysight Technologies (formerly Agilent) http://www .keysight.com/main/home.jspx.

[16] http://www.tek.com//?zct=US.

[17] F. G. Harris, On the use of windows for harmonic analysis with the discrete Fourier transform, Proc. IEEE 66, 51 (1978). 